\title{
JOINTLY QUASINORMAL ISOMETRIES
}

\author{
MARY EMBRY-WARDROP AND RICHARD J. FLEMING
}

\begin{abstract}
If $U$ and $V$ are isometries each of which commutes with $U^{*} V$ and $V^{*} U$, then a necessary and sufficient condition that $U$ and $V$ commute is that the ranges of $U$ and $V$ are equal. This result leads to the construction of a subnormal-valued analytic function which has no normal extension.
\end{abstract}

In [2] Globevnik and Vidav proved that if $f$ is an analytic function whose values are normal operators on a Hilbert space $X$, then the range of $f$ is abelian. In [1] Fleming and Jamison ask if this result is valid when the values of a function are subnormal or even quasinormal. A related question is whether an analytic subnormal-valued function has an extension to an analytic normal-valued function. The answer to each of these questions is no, as will be seen in Example 2.

A sufficient condition that the values of an analytic function $f$ be quasinormal is that $A\left(B^{*} C\right)=\left(B^{*} C\right) A$ whenever $A, B$ and $C$ are coefficients of $f$. If this condition holds and $A$ and $B$ are coefficients of $f$, then each of $A$ and $B$ commutes with each of $A^{*} A, A^{*} B, B^{*} A$ and $B^{*} B$, in which case we shall call $A$ and $B$ jointly quasinormal. For the simple analytic function $f(z)=A+z B$ we can now paraphrase the question in [1] by asking whether $A$ and $B$ commute when $A$ and $B$ are jointly quasinormal. The answer to this question is also no, as will be seen in Example 1.

The key to the answers of the above-mentioned questions is in the following theorem concerning isometries. The terminology used in the paper is as follows: $A$ is normal if $A$ commutes with $A^{*}$, quasinormal if $A$ commutes with $A^{*} A$, an isometry if $A^{*} A=I$, and a partial isometry if $A^{*} A$ is a projection. Basic facts concerning these special operators can be found in [3]. The range of an operator $A$ is denoted by $A(X)$.

THEOREM. If $U$ and $V$ are jointly quasinormal isometries, the following are equivalent:

(i) $U V=V U$,

(ii) $U V(X)=V U(X)$,

(iii) $U(X)=V(X)$.

ProOF. (i) $\Rightarrow$ (ii) trivially. To see that (ii) $\Rightarrow($ iii) note that if $U V(X)=V U(X)$ then $U^{*} U V(X)=U^{*} V U(X)$. Consequently, $V(X)=U\left(U^{*} V\right)(X)$ since $U$ is an isometry and $U$ and $V$ are jointly quasinormal. Thus, $V(X) \subset U(X)$ if $U V(X)=$ $V U(X)$ and by symmetry $U(X) \subset V(X)$ also. To see that (iii) $\Rightarrow(\mathrm{i})$ assume that $U(X)=V(X)$ or equivalently $U U^{*}=V V^{*}$ since $U$ and $V$ are (partial) isometries. Let $K=U V-V U$ and note that $K(X) \subset U(X)$ since $V(X) \subset U(X)$. Furthermore, $U^{*} K=U^{*} U V-U^{*} V U=V-U U^{*} V$ (since $U$ is an isometry and $U$ and $V$ are

Received by the editors May 13, 1985 and, in revised form, July 19, 1985.

1980 Mathematics Subject Classification. Primary 47B20. 
jointly quasinormal) $=V-V V^{*} V$ (since $\left.V V^{*}=U U^{*}\right)=0$ (since $V$ is an isometry). Therefore, $U^{*} K=0$, so that $K(X)$ is orthogonal to $U(X)$. We previously showed $K(X) \subset U(X)$ also. These two results imply that $K=0$ or that $U V=V U$, as desired. Q.E.D.

This theorem makes the task of constructing noncommuting jointly quasinormal operators easy. Alan Lambert first suggested the simple construction in Example 1.

EXAMPLE 1. Let $X$ be a Hilbert space with orthonormal basis $\left\{e_{n}: n=1,2, \ldots\right\}$. Let $U$ and $V$ be the isometries for which $U e_{n}=e_{2 n}$ and $V e_{n}=e_{2 n-1}$. $U$ and $V$ do not commute since $U V e_{1}=e_{2}$ and $V U e_{1}=e_{3}$. On the other hand, $U^{*} V=$ $V^{*} U=0$ since $U(X)$ and $V(X)$ are orthogonal. Since $U^{*} U=V^{*} V=I$, all of the commutation properties for the joint quasinormality of $U$ and $V$ are satisfied trivially.

EXAMPLE 2. Let $U$ and $V$ be the noncommuting jointly quasinormal isometries in Example 1 and define $f(z)=U+z V$ for each complex number $z$. Note that $f(z)^{*} f(z)=\left(1+|z|^{2}\right) I$ where $I$ is the identity operator, so that each value $f(z)$ is quasinormal, and consequently, subnormal. Thus we have an example of a subnormal-valued analytic function with nonabelian range. To see that this also provides us with a subnormal-valued analytic function which does not have a normal-valued analytic extension we need only recall that such an extension would have an abelian range [2]. This, of course, would force $f$ to have an abelian range.

We close with two observations. If $A$ and $B$ are jointly quasinormal operators with canonical polar decompositions $U P$ and $V Q$, respectively, then $U$ and $V$ are jointly quasinormal partial isometries. (The proof of this depends upon a rather lengthy, but elementary, algebraic computation.) Moreover, the jointly quasinormal operators $A$ and $B$ commute exactly when $U$ and $V$ commute. Secondly, it follows easily from the Theorem that when $U$ and $V$ are jointly quasinormal partial isometries, a necessary and sufficient condition for $U$ and $V$ to commute is that $U V(X)=V U(X)$. Thus, the general question of commutativity of quasinormal operators reduces to consideration of their partially isometric factors.

\section{REFERENCES}

1. R. J. Fleming and J. E. Jamison, Commutative ranges of analytic functions in Banach space, Proc. Amer. Math. Soc. 93 (1985), 48-59.

2. J. Globevnik and I. Vidav, A note on normal-operator-valued analytic functions, Proc. Amer. Math. Soc. 37 (1973), 619-621.

3. P. R. Halmos, A Hilbert space problem book, Van Nostrand, Princeton, N. J., 1967.

Department of Mathematics, Central Michigan University, Mt. Pleasant, MICHIGAN 48858 